\title{
Quality by Design towards Standardization of 3D Printed Bone Implants and scaffolds for Industry Translation
}

\author{
Daniel Martinez-Marquez ${ }^{1}$, Karan. Gulati², Ali. Mirnajafizadeh ${ }^{3}$ Christopher P. Carty $^{4,5,6}$, \\ Rodney Stewart ${ }^{1}$, Saso Ivanovski ${ }^{2}$ \\ ${ }^{1}$ School of Engineering and Built Environment, Griffith University \\ Gold Coast, Campus, Parklands Drive, QLD 4222 Australia \\ daniel.martinezmarquez@griffithuni.edu.au \\ r.stewart@griffith.edu.au \\ ${ }^{2}$ School of Dentistry, The University of Queensland \\ Herston, QLD, Australia \\ k.gulati@uq.edu.au; s.ivanovski@uq.edu.au \\ ${ }^{3}$ Molecular Cell Biomechanics Laboratory, University of Berkeley \\ Berkeley, California, USA \\ ali.mirnajafi@gmail.com \\ ${ }^{4}$ School of Allied Health Sciences, Menzies Health Institute Queensland, Griffith University \\ Gold Coast, QLD, Australia \\ ${ }^{5}$ Centre for Musculoskeletal Research, Menzies Health Institute Queensland, Griffith University \\ Gold Coast, QLD, Australia \\ ${ }^{6}$ Queensland Children's Gait Laboratory, Queensland Paediatric Rehabilitation Service, \\ Children's Health Queensland Hospital and Health Service \\ Brisbane, QLD, Australia. \\ c.carty@griffith.edu.au
}

\section{Extended Abstract}

3D printing is an emergent manufacturing technology recently being applied in the biomedical field for the development of custom bone implants and scaffolds. Moreover, new technologies currently in research such as motion capture and nano engineered surfaces can be fully integrated with 3D printing to greatly enhance custom bone implants performance. However, successful industry transformation to this new design and manufacturing approach requires concurrent multi-disciplinary collaboration, and a robust and flexible quality management framework to integrate different technologies. This later change enabler is the focus of this study.

While, a number of good quality frameworks have been developed in recent decades, they are centred on the traditional context of standardised manufacturing techniques, which are not suitable for 3D printing technology and customized products [1]. Moreover, most emergent biomedical technologies have to face numerous changes, iterations, and evaluations to achieve final product concept and design [2]. However, biomedical research requires expensive and advanced technologies that drain most of its funding without even reaching pre-clinical and clinical studies to demonstrate product safety, and obtain clinical approval [3,4]. This gap is known as the "Valley of Death" and is where most ventures die [4].

The advent of 3D printing, emergent technologies, and the prospects for mass customisation provides significant market opportunities, but also presents a serious challenge to regulatory bodies around the world for managing and assuring product quality and safety. Before 3D printed bone implants and the associated emergent technologies can gain traction, industry stakeholders, such as regulators, clients, medical practitioners, insurers, lawyers, and manufacturers, would all require a high degree of confidence that customised manufacturing can achieve the same quality outcomes as standardised manufacturing.

The Quality by Design (QbD) approach can ensure that products are designed and manufactured correctly from the beginning without errors, avoiding trial-and-error studies to also accelerate research timelines and reduce development costs [5-7]. Furthermore, QbD can pave the way for technologies and processes that have the potential to be scalable and reach 
commercial stages. QbD encourages process and product understanding to support innovation and efficiency in product development, and to meet FDA regulatory requirements [8].

Bringing the concept of QbD into the context of custom 3D printed bone implants and scaffold, this study explores the technologies and activities involved in the design and fabrication of these products. Therefore, the purpose of this research is to provide a flexible tool which can be used by both researchers and industry through the adaptation of the QbD approach for the initial design stages of custom 3D printed bone implants, and nano engineered surfaces with titania nanotubes (TNTs) for therapeutic bone/dental implants. For these we considered the ICHQ8(R2) guidelines [9] and existing quality risk management tools.

This is a qualitative exploratory research study with a constructive research approach aimed to produce innovative solutions to practical problems in a heuristic manner, followed by validating the solution afterwards [35]. The data collection and validation of this study involved various systematic searches in different scientific databases, an online survey, and face to face interviews with pertinent researchers, industry experts, and medical practitioners from different fields related to medical device development, 3D bone printed implants, motion capture, bone biology, tissue engineering, orthopaedic surgery, bone biomechanics, computational neuromuscular modelling, and nano engineered implants.

Research outcomes include: The identification of the main applications and benefits of the QbD approach in different research studies; The development of a comprehensive design and fabrication process flow diagram of 3D bone printed implants; A list of 86 categorised quality risks and 178 effects associated with the design and fabrication processes of 3D bone printed implants; The identification of the TNTs' characteristics necessary for commercial purposes; The identification and ranking of the influence of TNTs characteristics on the critical quality attributes of nano engineered surfaces using the Quality Function Deployment method.

\section{References}

[1] M. Monzón, Z. Ortega, A. Martínez, and F. Ortega, "Standardization in additive manufacturing: activities carried out by international organizations and projects," The International Journal of Advanced Manufacturing Technology, vol. 76, pp. 1111-1121, 2015.

[2] U. S. Food and Drug Administration, Medical device innovation initiative white paper: CDRH innovation initiative, vol. 2016, 2011.

[3] J. D. Sipe, "Tissue Engineering and Reparative Medicine," Annals of the New York Academy of Sciences, vol. 961, pp. $1-9,2002$.

[4] S. J. Hollister, "Scaffold engineering: a bridge to where?," Biofabrication, vol. 1, p. 012001, 2009.

[5] N. V. R. Naidu, K. M. Babu, G. Rajendra, and I. ebrary, Total quality management, vol. 1. New Delhi: New Age International (P) Ltd., Publishers, 2006.

[6] X. Y. Lawrence, "Pharmaceutical quality by design: product and process development, understanding, and control," Pharmaceutical research, vol. 25, pp. 781-791, 2008.

[7] M. W. Krucoff, R. G. Brindis, P. K. Hodgson, M. J. Mack, and J. D. R. Holmes, "Medical device innovation: prospective solutions for an ecosystem in crisis. Adding a professional society perspective," JACC Cardiovascular interventions, vol. 5, p. 790, 2012.

[8] J. N. Sangshetti, M. Deshpande, Z. Zaheer, D. B. Shinde, and R. Arote, "Quality by design approach: Regulatory need," Arabian Journal of Chemistry, 2014.

[9] ICH Harmonised Tripartite Guideline, "Pharmaceutical development Q8 (R2)," ICH Steering Committee, Step, vol. 4, 2009. 